Bull. Austral. Math. Soc.

VOL. 60 (1999) [73-80]

\title{
HIRSCHHORN'S IDENTITIES
}

\author{
Paul Hammond, Richard Lewis and Zhi-Guo LiU
}

\begin{abstract}
We prove a general identity between power series and use this identity to give proofs of a number of identities proposed by M.D. Hirschhorn. We also use the identity to give proofs of a well-known result of Jacobi, the quintuple-product identity and Winquist's identity.
\end{abstract}

\section{INTRODUCTION}

We suppose throughout that $q$ is a complex number with $|q|<1$; this condition ensures that all the sums and products that appear here converge.

In January 1998, Mike Hirschhorn conjectured (written communication) that

(H)

$$
\begin{aligned}
& \sum_{n \in \mathbb{Z}} q^{3 n^{2}} \sum_{n \in \mathbb{Z}} q^{3 n^{2}+n}+q \sum_{n \in \mathbb{Z}} q^{3 n^{2}+2 n} \sum_{n \in \mathbb{Z}} q^{3 n^{2}+3 n}=\frac{\left(q^{3}, q^{6} ; q^{6}\right)_{\infty}^{2}}{\left(q, q^{5} ; q^{6}\right)_{\infty}^{2}} \\
& \sum_{n \in \mathbb{Z}} q^{5 n^{2}} \sum_{n \in \mathbb{Z}} q^{5 n^{2}+n}+q^{2} \sum_{n \in \mathbb{Z}} q^{5 n^{2}+4 n} \sum_{n \in \mathbb{Z}} q^{5 n^{2}+5 n}=\frac{\left(q^{4}, q^{5}, q^{6}, q^{10} ; q^{10}\right)_{\infty}^{2}}{\left(q^{2}, q^{3}, q^{7}, q^{8} ; q^{10}\right)_{\infty}^{2}} \\
& \sum_{n \in \mathbb{Z}} q^{5 n^{2}} \sum_{n \in \mathbb{Z}} q^{5 n^{2}+3 n}+q \sum_{n \in \mathbb{Z}} q^{5 n^{2}+2 n} \sum_{n \in \mathbb{Z}} q^{5 n^{2}+5 n}=\frac{\left(q^{2}, q^{5}, q^{8}, q^{10} ; q^{10}\right)_{\infty}^{2}}{\left(q, q^{4}, q^{6}, q^{9} ; q^{10}\right)_{\infty}^{2}} \\
& \sum_{n \in \mathbb{Z}} q^{7 n^{2}+n} \sum_{n \in \mathbb{Z}} q^{7 n^{2}+2 n}+q^{2} \sum_{n \in \mathbb{Z}} q^{7 n^{2}+5 n} \sum_{n \in \mathbb{Z}} q^{7 n^{2}+6 n}=\frac{\left(q^{6}, q^{7}, q^{7}, q^{8}, q^{14}, q^{14} ; q^{14}\right)_{\infty}}{\left(q^{2}, q^{3}, q^{5}, q^{9}, q^{11}, q^{12} ; q^{14}\right)_{\infty}} \\
& \sum_{n \in \mathbb{Z}} q^{7 n^{2}+2 n} \sum_{n \in \mathbb{Z}} q^{7 n^{2}+3 n}+q \sum_{n \in \mathbb{Z}} q^{7 n^{2}+4 n} \sum_{n \in \mathbb{Z}} q^{7 n^{2}+5 n}=\frac{\left(q^{2}, q^{7}, q^{7}, q^{12}, q^{14}, q^{14} ; q^{14}\right)_{\infty}}{\left(q, q^{3}, q^{4}, q^{10}, q^{11}, q^{13} ; q^{14}\right)_{\infty}} \\
& \sum_{n \in \mathbb{Z}} q^{7 n^{2}+n} \sum_{n \in \mathbb{Z}} q^{7 n^{2}+4 n}+q \sum_{n \in \mathbb{Z}} q^{7 n^{2}+3 n} \sum_{n \in \mathbb{Z}} q^{7 n^{2}+6 n}=\frac{\left(q^{4}, q^{7}, q^{7}, q^{10}, q^{14}, q^{14} ; q^{14}\right)_{\infty}}{\left(q, q^{5}, q^{6}, q^{8}, q^{9}, q^{13} ; q^{14}\right)_{\infty}}
\end{aligned}
$$

Received 21st December, 1998

Copyright Clearance Centre, Inc. Serial-fee code: 0004-9729/99 \$A2.00+0.00. 
and asked for proofs. Here,

$$
\left(q^{a_{1}}, q^{a_{2}}, \ldots, q^{a_{k}} ; q^{n}\right)_{\infty}:=\left(q^{a_{1}} ; q^{n}\right)_{\infty}\left(q^{a_{2}} ; q^{n}\right)_{\infty} \ldots\left(q^{a_{k}} ; q^{n}\right)_{\infty}
$$

where

$$
(z ; q)_{\infty}:=\prod_{n=0}^{\infty}\left(1-z q^{n}\right)
$$

We also write

$$
[z ; q]_{\infty}:=(z ; q)_{\infty}\left(z^{-1} q ; q\right)_{\infty}
$$

and

$$
\left[z_{1}, z_{2}, \ldots, z_{n} ; q\right]_{\infty}:=\left[z_{1} ; q\right]_{\infty}\left[z_{2} ; q\right]_{\infty} \cdots\left[z_{n} ; q\right]_{\infty}
$$

for $z_{1}, z_{2}, \ldots, z_{n} \neq 0$. (The function $[z ; q]_{\infty}$ is a close relative of the Jacobian theta functions. Indeed, with $z=e^{2 \pi i x}, q=e^{2 \pi i \tau}$, we have $\theta_{1}(\pi x \mid \tau)=i q^{1 / 8} z^{-1 / 2}(q ; q)_{\infty}[z ; q]_{\infty}$, see $[10$, p.470]). With this notation, note that, as a function of $z$,

$$
[z ; q]_{\infty} \text { has simple zeros at } z=q^{k}, \text { for } k \in \mathbb{Z}
$$

an essential singularity at $z=0$ and no other zeros and no poles. We have the simple and easily verifiable properties:

$$
\begin{aligned}
{\left[z^{-1} ; q\right]_{\infty}=-z^{-1}[z ; q]_{\infty} } & =[z q ; q]_{\infty} \\
{\left[z, z q, \cdots, z q^{k-1} ; q^{k}\right]_{\infty} } & =[z ; q]_{\infty} \\
{\left[z, \zeta z, \ldots, \zeta^{k-1} z ; q\right]_{\infty} } & =\left[z^{k} ; q^{k}\right]_{\infty}
\end{aligned}
$$

where $\zeta=e^{2 \pi i / k}$, and we note that it follows from (1.3) that

$$
\left[z^{-1} q ; q\right]_{\infty}=[z ; q]_{\infty}
$$

Jacobi's well-known triple product identity (see [4, Section 19.8.1] or [6] for an analytic. respectively, combinatorial proof) states that

$$
(q ; q)_{\infty}[z ; q]_{\infty}=\sum_{n \in \mathbb{Z}} z^{n} q^{n(n-1) / 2}
$$

With this notation (1.1), it may be readily checked that all the identities $(\mathrm{H})$ are 
instances of:

$$
\begin{aligned}
\sum_{n \in \mathbb{Z}} q^{M n^{2}+(x-t) n} \sum_{n \in \mathbb{Z}} q^{M n(n-1)+(x+t) n}+q^{t} \sum_{n \in \mathbb{Z}} q^{M n^{2}+(x+t) n} \sum_{n \in \mathbb{Z}} q^{M n(n-1)+(x-t) n} \\
=\frac{\left[q^{2 t}, q^{2 x}, q^{M} ; q^{2 M}\right]_{\infty}\left(q^{2 M} ; q^{2 M}\right)_{\infty}}{\left[q^{t}, q^{M+t}, q^{x}, q^{M+x} ; q^{2 M}\right]_{\infty}}
\end{aligned}
$$

which, by (1.4) and (1.5),

$$
=\left[-q^{t},-q^{x} ; q^{M}\right]_{\infty}\left(q^{M} ; q^{M}\right)_{\infty}^{2}
$$

and this, by (1.7),

$$
=\sum_{n \in \mathbb{Z}} q^{M n(n-1) / 2+t n} \sum_{n \in \mathbb{Z}} q^{M n(n-1) / 2+x n}
$$

and it is the general identity (1.8) that we prove in Theorem 2 below.

\section{The PRoOF of The IDENTITIES}

Suppose $q \in \mathbb{C} \backslash\{0\}$ (with $|q|<1$ ). Following [2], say that $z_{0}, w_{0} \in \mathbb{C} \backslash\{0\}$ are equivalent if $z_{0}=q^{n} w_{0}$, for some $n \in \mathbb{Z}$.

LEMma. Suppose $f: \mathbb{C} \backslash\{0\} \rightarrow \mathbb{C}$ is analytic save for some isolated simple poles and suppose that $f$ satisfies

$$
f(q z)=q^{-1} f(z)
$$

for $z \neq 0$. If $\mathcal{P}$ is a complete set of inequivalent poles of $f(\mathcal{P}$ is necessarily finite), then

$$
\sum_{z \in \mathcal{P}} \operatorname{res}(f ; z)=0
$$

Proof: First note that, if $f: \mathbb{C} \backslash\{0\} \rightarrow \mathbb{C}$ satisfies (2.1) and if $z_{0}$ and $w_{0}$ are equivalent points, $z_{0}=q^{n} w_{0}$, say, then $w_{0}$ is a simple pole of $f$ if and only if $z_{0}$ is a simple pole of $f$ and

$$
\begin{aligned}
\operatorname{res}\left(f ; z_{0}\right) & =\lim _{z \rightarrow z_{0}}\left(z-z_{0}\right) f(z) \\
& =\lim _{w \rightarrow w_{0}}\left(q^{n} w-q^{n} w_{0}\right) f\left(q^{n} w\right) \\
& =\lim _{w \rightarrow w_{0}} q^{n}\left(w-w_{0}\right) q^{-n} f(w) \\
& =\lim _{w \rightarrow w_{0}}\left(w-w_{0}\right) f(w)=\operatorname{res}\left(f ; w_{0}\right)
\end{aligned}
$$


Thus the choice of $\mathcal{P}$ is immaterial in evaluating the sum at (2.2). Now let $\mathcal{A}:=\{z$ : $r|q| \leqslant|z|<r\} \quad(r>0)$ be a half-open annulus in $\mathbb{C}$, centred at the origin. So $\mathcal{A}$ includes its inner boundary circle, $S_{2}=\{w:|w|=r|q|\}$, but not its outer boundary circle $S_{1}=\{z:|z|=r\}$. The non-zero poles of $f$ are isolated and so we can, changing $r$, if necessary, suppose that neither $S_{1}$ nor $S_{2}$ contains any pole or zero of $f$. It is plain that every $z \in \mathbb{C} \backslash\{0\}$ is equivalent to a unique point in $\mathcal{A}$ and that distinct points in $\mathcal{A}$ are inequivalent and so we may take $\mathcal{P}$ to be the set of poles of $f$ in $\mathcal{A}$. Now

$$
\sum_{z \in \mathcal{P}} \operatorname{res}(f ; z)=\frac{1}{2 \pi i} \int_{S_{1}} f(z) d z-\frac{1}{2 \pi i} \int_{S_{2}} f(w) d w
$$

But if $w \in S_{2}$, then $w=q z$, where $z \in S_{1}$. We have $d w=q d z$ and $f(w)=f(q z)=$ $q^{-1} f(z)$ and so

$$
\sum_{z \in \mathcal{P}} \operatorname{res}(f ; z)=\frac{1}{2 \pi i} \int_{S_{1}} f(z) d z-\frac{1}{2 \pi i} \int_{S_{1}} q^{-1} f(z) q d z=0
$$

(This Lemma is really a disguised form of a well-known property of elliptic functions; namely, that the sum of the residues of an elliptic function at its poles in a period parallelogram is zero.)

TheOREM 1. Suppose $a_{1}, a_{2}, \ldots, a_{n} ; b_{1}, b_{2}, \ldots, b_{n} \in \mathbb{C} \backslash\{0\}$ satisfy:

(i) $a_{i}$ and $a_{j}$ are inequivalent, for $i \neq j$.

(ii) $a_{1} a_{2} \ldots a_{n}=b_{1} b_{2} \ldots b_{n}$. Then

$$
\sum_{i=1}^{n} \frac{\prod_{j=1}^{n}\left[a_{i} b_{j}^{-1} ; q\right]_{\infty}}{\prod_{j=1, j \neq i}^{n}\left[a_{i} a_{j}^{-1} ; q\right]_{\infty}}=0 .
$$

Proof: Define

$$
\mathcal{F}(z)=\mathcal{F}(z ; q):=\frac{\prod_{j=1}^{n}\left[z b_{j}^{-1} ; q\right]_{\infty}}{z \prod_{j=1}^{n}\left[z a_{j}^{-1} ; q\right]_{\infty}}
$$

Condition (i) shows that $\mathcal{F}$ has only simple poles in $\mathbb{C} \backslash\{0\}$ and that $\left\{a_{1}, a_{2}, \ldots, a_{n}\right\}$ is a complete set of inequivalent poles. Taking into account (1.3), condition (ii) shows that (2.1) is satisfied. Now the residue of $\mathcal{F}$ at $a_{i}$ is

$$
\operatorname{res}\left(\mathcal{F} ; a_{i}\right)=\lim _{z \rightarrow a_{i}}\left(z-a_{i}\right) \mathcal{F}(z)
$$




$$
\begin{aligned}
& =\frac{\prod_{j=1}^{n}\left[a_{i} b_{j}^{-1} ; q\right]_{\infty}}{a_{i} \prod_{j=1, j \neq i}^{n}\left[a_{i} a_{j}^{-1} ; q\right]_{\infty}} \lim _{z \rightarrow a_{i}} \frac{\left(z-a_{i}\right)}{\left[z a_{i}^{-1} ; q\right]_{\infty}} \\
& =\frac{\prod_{j=1}^{n}\left[a_{i} b_{j}^{-1} ; q\right]_{\infty}}{a_{i} \prod_{j=1, j \neq i}^{n}\left[a_{i} a_{j}^{-1} ; q\right]_{\infty}} \frac{1}{(q ; q)_{\infty}^{2}} \lim _{z \rightarrow a_{i}} \frac{\left(z-a_{i}\right)}{\left(1-z a_{i}^{-1}\right)} \\
& =-\frac{1}{(q ; q)_{\infty}^{2}} \frac{\prod_{j=1}^{n}\left[a_{i} b_{j}^{-1} ; q\right]_{\infty}}{\prod_{j=1, j \neq i}^{n}\left[a_{i} a_{j}^{-1} ; q\right]_{\infty}} .
\end{aligned}
$$

(2.3) now follows from (2.2).

Theorem 1 appears as $[8,(7.4 .3)]$, though no proof is given. The author states that it is a consequence of the 'general theorem' on (Weierstrass) sigma functions and refers to [10, p.451, exercise 3], where again no proof is given. The proof given here appeared in [7]. We can now prove

Theorem 2. If $x, t, M \in \mathbb{Z}$ and $0<t<M$, then

$$
\begin{aligned}
& \sum_{n \in \mathbb{Z}} q^{M n^{2}+(x-t) n} \sum_{n \in \mathbb{Z}} q^{M n(n-1)+(x+t) n}+q^{t} \sum_{n \in \mathbb{Z}} q^{M n^{2}+(x+t) n} \sum_{n \in \mathbb{Z}} q^{M n(n-1)+(x-t) n} \\
& \quad=\sum_{n \in \mathbb{Z}} q^{M n(n-1) / 2+x n} \sum_{n \in \mathbb{Z}} q^{M n(n-1) / 2+t n} .
\end{aligned}
$$

Proof: Take $n=3$ in Theorem 1 and $q^{2 M}$ for $q$ and set

$$
\left(a_{1}, a_{2}, a_{3} ; b_{1}, b_{2}, b_{3}\right)=\left(1, q^{2 t}, q^{M+t} ;-q^{x+t},-q^{M-x+t}, q^{t}\right) .
$$

The hypotheses of Theorem 1 are satisfied and (2.3) gives

$$
\begin{gathered}
\frac{\left[-q^{-(x+t)},-q^{-(M-x+t)}, q^{-t} ; q^{2 M}\right]_{\infty}}{\left[q^{-2 t}, q^{-(M+t)} ; q^{2 M}\right]_{\infty}}+\frac{\left[-q^{-(x-t)},-q^{-(M-x-t)}, q^{t} ; q^{2 M}\right]_{\infty}}{\left[q^{2 t}, q^{-(M-t)} ; q^{2 M}\right]_{\infty}} \\
+\frac{\left[-q^{M-x},-q^{x}, q^{M} ; q^{2 M}\right]_{\infty}}{\left[q^{(M+t)}, q^{(M-t)} ; q^{2 M}\right]_{\infty}}=0
\end{gathered}
$$

We use (1.3) to write this as

$$
\begin{aligned}
&-\frac{\left[-q^{x+t},-q^{M-x+t}, q^{t} ; q^{2 M}\right]_{\infty}}{\left[q^{2 t}, q^{M+t} ; q^{2 M}\right]_{\infty}}-q^{t} \frac{\left[-q^{x-t},-q^{M-x-t}, q^{t} ; q^{2 M}\right]_{\infty}}{\left[q^{2 t}, q^{M-t} ; q^{2 M}\right]_{\infty}} \\
&+\frac{\left[-q^{M-x},-q^{x}, q^{M} ; q^{2 M}\right]_{\infty}}{\left[q^{(M+t)}, q^{(M-t)} ; q^{2 M}\right]_{\infty}}=0
\end{aligned}
$$


or

$$
\left[-q^{x+t},-q^{M-x+t} ; q^{2 M}\right]_{\infty}+q^{t}\left[-q^{x-t},-q^{M-x-t} ; q^{2 M}\right]_{\infty}=\frac{\left[-q^{x},-q^{M+x}, q^{2 t}, q^{M} ; q^{2 M}\right]_{\infty}}{\left[q^{t}, q^{M+t} ; q^{2 M}\right]_{\infty}}
$$

since, by (1.6), $\left[q^{M-x} ; q^{2 M}\right]_{\infty}=\left[q^{M+x} ; q^{2 M}\right]_{\infty}$. Now multiply each side by $\left(q^{2 M} ; q^{2 M}\right)_{\infty}^{2}$ and use (1.4) and (1.5) to get

$$
\begin{array}{r}
{\left[-q^{x+t},-q^{M-x+t} ; q^{2 M}\right]_{\infty}\left(q^{2 M} ; q^{2 M}\right)_{\infty}^{2}+q^{t}\left[-q^{x-t},-q^{M-x-t} ; q^{2 M}\right]_{\infty}\left(q^{2 M} ; q^{2 M}\right)_{\infty}^{2}} \\
=\left[-q^{x} ; q^{M}\right]_{\infty}\left[-q^{t} ; q^{M}\right]_{\infty}\left(q^{M} ; q^{M}\right)_{\infty}^{2}
\end{array}
$$

which, as we pointed out earlier, translates by way of (1.7) into (2.4).

Now (2.4) appears in Hirschhorn's work (it is [5, (2.1)] after applying the triple product identity), so he had already proved the identities $(\mathrm{H})$ he was asking about as well as their generalisation (1.8). His proof of (2.4) is very different from that given here. He multiplies together two copies of the triple product identity and sorts the resulting equation into even and odd powers of $q$. (2.4) also appeared in [3] with more or less the same (that is, Hirschhorn's) proof.

\section{THREE MORE IDENTITIES}

Theorem 1 can plainly be used to generate a large number of identities of the same type as (2.4). (infinitely many, of course). We shall give three more applications of Theorem 1.

1. Our first application is another of Jacobi's marvellous identities. Taking $n=3$ and $q^{2}$ in place of $q$ and with $\left(a_{1}, a_{2}, a_{3} ; b_{1}, b_{2}, b_{3}\right)=\left(1,-q,-q^{2} ; q, q, q\right)$, Theorem 1 gives

$$
\frac{\left[q^{-1} ; q^{2}\right]_{\infty}^{3}}{\left[-q^{-1},-q^{-2} ; q^{2}\right]_{\infty}}+\frac{\left[-1 ; q^{2}\right]_{\infty}^{3}}{\left[-q, q^{-1} ; q^{2}\right]_{\infty}}+\frac{\left[-q ; q^{2}\right]_{\infty}^{3}}{\left[-q^{2}, q ; q^{2}\right]_{\infty}}=0
$$

from which, with (1.3) and (1.6), we get

$$
-\left[q ; q^{2}\right]_{\infty}^{4}-q\left[-1 ; q^{2}\right]_{\infty}^{4}+\left[-q ; q^{2}\right]_{\infty}^{4}=0
$$

Noting that $\left[-1 ; q^{2}\right]_{\infty}=2\left(-q^{2} ; q^{2}\right)_{\infty}^{2}$, this translates into Jacobi's identity

$$
\left\{\prod_{n=1}^{\infty}\left(1-q^{2 n-1}\right)\right\}^{8}+16 q\left\{\prod_{n=1}^{\infty}\left(1+q^{2 n}\right)\right\}^{8}=\left\{\prod_{n=1}^{\infty}\left(1+q^{2 n-1}\right)\right\}^{8}
$$

(According to [10, p.470, footnote], Jacobi said of this identity that it was an 'aequatio identica satis abstrusa'.) 
2. We now use Theorem 1 to derive the quintuple product identity [2], which in one form states:

$$
\frac{\left[z^{2} ; q\right]_{\infty}}{[z ; q]_{\infty}}(q ; q)_{\infty}=\left\{\left[z^{3} q ; q^{3}\right]_{\infty}+z\left[z^{-3} q ; q^{3}\right]_{\infty}\right\}\left(q^{3} ; q^{3}\right)_{\infty}
$$

If we set $n=3, \omega=e^{2 \pi i / 3}$ and $\left(a_{1}, a_{2}, a_{3} ; b_{1}, b_{2}, b_{3}\right)=\left(z, z^{-1}, q ; q^{1 / 3}, q^{1 / 3} \omega, q^{1 / 3} \omega^{-1}\right)$ in Theorem 1, (2.3) becomes

$$
\begin{gathered}
\frac{\left[z q^{-1 / 3}, z q^{-1 / 3} \omega^{-1}, z q^{-1 / 3} \omega ; q\right]_{\infty}}{\left[z^{2}, z q^{-1} ; q\right]_{\infty}}+\frac{\left[z^{-1} q^{-1 / 3}, z^{-1} q^{-1 / 3} \omega^{-1}, z^{-1} q^{-1 / 3} \omega ; q\right]_{\infty}}{\left[z^{-2}, z^{-1} q^{-1} ; q\right]_{\infty}} \\
+\frac{\left[q^{2 / 3}, q^{2 / 3} \omega^{-1}, q^{2 / 3} \omega ; q\right]_{\infty}}{\left[z^{-1} q, z q ; q\right]_{\infty}}=0,
\end{gathered}
$$

which, with the help of (1.3) and (1.5), gives (3.2).

3. Finally, Winquist's Identity [9], namely:

$$
\begin{gathered}
{[x ; q]_{\infty}[y ; q]_{\infty}\left[x y^{-1} ; q\right]_{\infty}[x y ; q]_{\infty}(q ; q)_{\infty}^{2}=x y^{-1}\left[y^{3} ; q^{3}\right]_{\infty}} \\
\left\{x\left[q x^{-3} ; q^{3}\right]_{\infty}-\left[q x^{3} ; q^{3}\right]_{\infty}\right\}\left(q^{3} ; q^{3}\right)_{\infty}^{2}-\left[x^{3} ; q^{3}\right]_{\infty}\left\{y\left[q y^{-3} ; q^{3}\right]_{\infty}-\left[q y^{3} ; q^{3}\right]_{\infty}\right\}\left(q^{3} ; q^{3}\right)_{\infty}^{2}
\end{gathered}
$$

also follows from Theorem 1, where now we take $n=6$ and

$$
\begin{aligned}
\left(a_{1}, a_{2}, a_{3}, a_{4}, a_{5}, a_{6} ; b_{1}, b_{2}, b_{3}, b_{4}, b_{5}, b_{6}\right) & \\
& =\left(x, x^{-1}, y, y^{-1}, \omega, \omega^{-1} ; q^{1 / 3}, q^{1 / 3} \omega, q^{1 / 3} \omega^{-1},-1,-q^{-1 / 2}, q^{-1 / 2}\right)
\end{aligned}
$$

We omit the details.

\section{Conclusion}

We have seen that Theorem 1 is very powerful and has many applications. A variant of this Theorem was used by Atkin and Swinnerton-Dyer to prove the Dyson conjectures on the ranks of partitions [2] and, in [7], the Theorem was again used to prove some other theorems about ranks. For these reasons, it would be very interesting to have a bijective/combinatorial proof of Theorem 1; such a proof might well lead to a bijective/combinatorial proof of the rank theorems, whose statements are, after all, combinatorial in nature. But even the formulation of a such a version of Theorem 1 seems fraught with difficulties.

Note that, in Theorem 1, the parameters $a_{1}, a_{2}, \ldots, a_{n}$ are required to be inequivalent. This condition ensures that the function $\mathcal{F}$ defined in the proof of Theorem 1 
has only simple poles, but what would we get if some of the $a_{i}$ were equivalent and so then the function $\mathcal{F}$ has poles of order $\geqslant 2$ ? Now the function

$$
\mathcal{G}(z)=\frac{[a z, b z, c z ; q]_{\infty}}{z[z, z, a b c z ; q]_{\infty}}
$$

satisfies (2.1) and so

$$
\operatorname{res}(\mathcal{G} ; 1)+\operatorname{res}\left(\mathcal{G} ;(a b c)^{-1}\right)=0
$$

It turns out that (4.1) leads to a striking identity relating a theta product and a sum of Lambert series from which many theorems on sums of squares may be deduced. A paper on this identity is in preparation [1].

\section{REFERENCES}

[1] G.E. Andrews, R.P. Lewis and Z-G. Liu, 'An Identity relating a theta function and a sum of Lambert series', (in preparation).

[2] A.O.L. Atkin and P. Swinnerton-Dyer, 'Some properties of partitions', Proc. London Math. Soc. (3) 4 (1954), 84-106.

[3] J.A. Ewell, 'Arithmetical consequences of a sextuple product identity', Rocky Mountain J. Math. 25 (1995), 1287-1293.

[4] G.H. Hardy and E.M. Wright, The theory of numbers, (3rd edition (Oxford University Press, 1954).

[5] M.D. Hirschhorn, 'A simple proof of Jacobi's four-square theorem', J. Austral. Math. Soc. Ser. A 32 (1982), 61-67.

[6] R.P. Lewis, 'A combinatorial proof of the triple product identity', Amer. Math. Monthly 91 (1984), 420-423.

[7] R.P. Lewis, 'On the ranks of partitions modulo 9', Bull. London Math. Soc. 23 (1991), 417-421.

[8] L.J. Slater, Generalized hypergeometric functions (Cambridge University Press, Cambridge, U.K., 1966).

[9] L. Winquist, 'An elementary proof of $p(11 m+6) \equiv 0(\bmod 11)$ ', J. Comb. Theory 6 (1969), 56-59.

[10] E.T. Whittaker and G.N. Watson, $A$ course of modern analysis, (4th edition) (Cambridge University Pess, New York, 1950).

SMS

The University of Sussex

Brighton BN1 9QH

United Kingdom 